\title{
New insights into altered gene regulation in schizophrenia
}

levels of RNA
editing were
increased
in the
dorsolateral
prefrontal
cortex and
the anterior
cingulate
cortex

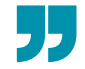

Two new studies, published in

Nature Communications and Nature

Neuroscience, provide evidence that the pathophysiology of schizophrenia involves changes in the regulation of gene expression at multiple levels. The key findings include RNA editing alterations and sex-specific gene expression signatures in individuals with schizophrenia.

In the first study, Jari Tiihonen and colleagues analysed gene and protein expression profiles in neurons derived from induced pluripotent stem cells (iPSCs). "We were interested in studying sex-specific pathophysiology related to gene and protein expression, as a recent study found that the pathophysiology of depression differs between men and women," explains Tiihonen. "To minimize the disease-irrelevant noise between affected and healthy individuals, we studied monozygotic twin pairs that were discordant for schizophrenia."

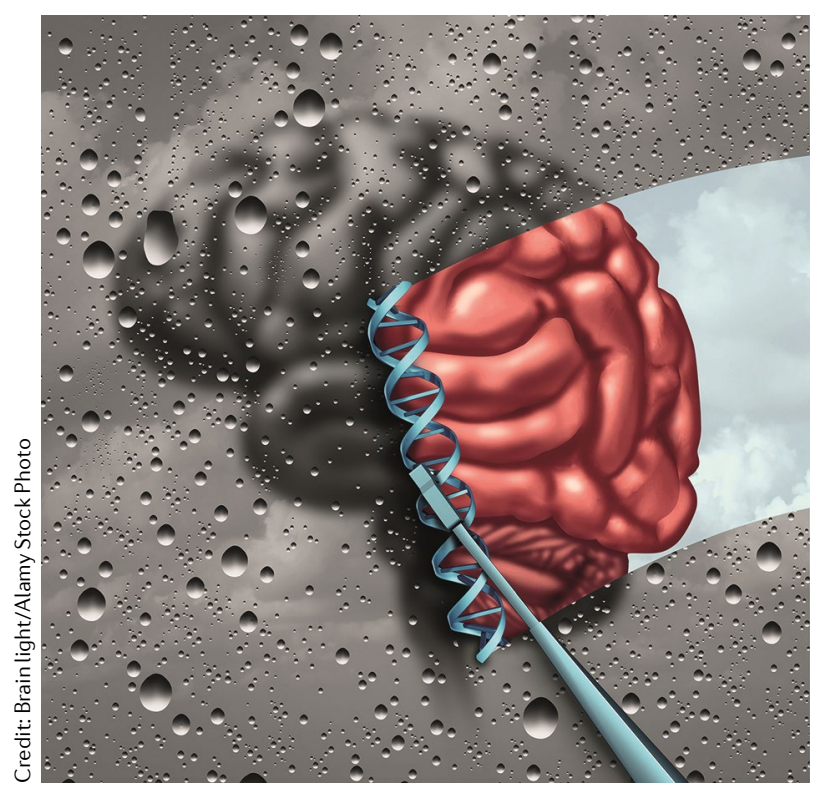

Tiihonen et al. analysed iPSCderived neurons from six pairs of monozygotic twins, each of which contained only one twin with schizophrenia, as well as from six unrelated age-matched controls. Schizophrenia was found to be associated with downregulation of pathways involved in glycosaminoglycan metabolism and upregulation of pathways involved in neurotransmitter catabolism and GABAergic synaptic function. However, the schizophrenia-specific gene and protein expression signatures were not the same in men and women.

"Although only $12 \%$ of all genes were expressed differentially between healthy men and women, up to $61 \%$ of the schizophrenia-related genes were sex-specific," reports Tiihonen. "This novel finding suggests that the pathophysiology differs between males and females, and may explain why schizophrenia manifests after adolescence when the expression of many sex-specific genes changes."

In the second study, Michael Breen, Joseph Buxbaum and colleagues explored the phenomenon of adenosine-to-inosine (A-to-I) RNA editing in the brains of people with schizophrenia. A-to-I RNA editing is a post-transcriptional event in which adenosine nucleotides are converted into inosine, thereby altering the sequence of the protein that is encoded by the RNA.

"Many RNA editing sites are located in genes involved in neuronal maintenance and development, and differences in editing levels have been associated with various neurological disorders, but have not previously been examined on a genome-wide scale," comments Breen. Buxbaum adds "we used RNA-sequencing data generated from the CommonMind
Consortium, which is a unique public-private consortium with the largest existing collection of collaborating brain banks of autopsied individuals with and without schizophrenia."

The researchers found that overall levels of RNA editing were increased in the dorsolateral prefrontal cortex and the anterior cingulate cortex in individuals with schizophrenia. However, analysis at the individualgene level revealed a more complex picture in these brain regions, with some RNAs showing reduced levels and others showing increased levels of editing.

Some of the underedited RNAs encoded proteins that have been implicated in schizophrenia, including glutamate receptors and postsynaptic density proteins. RNAs encoding molecules that are required for mitochondrial protein translation were prominent among the overedited RNAs - a finding that might help to explain reports of mitochondrial dysfunction in schizophrenia. Moreover, $~ 5 \%$ of schizophrenia risk loci previously identified in genome-wide association studies co-localized with RNA editing sites, suggesting that these loci mediate their detrimental effects through altered RNA editing.

"The next major question for this work, and for the RNA editing field, is: are these candidate schizophrenia-related editing sites functional and, if so, what are their functions?" concludes Breen. "This work generates inroads for examining the potential for targeting the editing sites, the respective protein or even the RNA editing-related pathway as a novel therapeutic approach for schizophrenia, and possibly other related disorders."

Heather Wood

ORIGINAL ARTICLES Tiihonen, J. et al. Sex-specific transcriptional and proteomic signatures in schizophrenia. Nat. Commun. 10, 3933 (2019) | Breen, M. S. et al. Global landscape and genetic regulation of RNA editing in cortical samples from individuals with schizophrenia. Nat. Neurosci. 22, 1402-1412 (2019) 